\title{
Les médiations du désir dans Les Soleils des indépendances d'Ahmadou Kourouma
}

\section{Alexandre Calvanese}

\section{(2) OpenEdition}

Journals

Édition électronique

URL : http://journals.openedition.org/rief/236

DOI : 10.4000/rief.236

ISSN : 2240-7456

Éditeur

Seminario di filologia francese

Référence électronique

Alexandre Calvanese, «Les médiations du désir dans Les Soleils des indépendances d'Ahmadou Kourouma », Revue italienne d'études françaises [En ligne], 3 | 2013, mis en ligne le 15 décembre 2013, consulté le 19 avril 2019. URL : http://journals.openedition.org/rief/236 ; DOI : 10.4000/rief.236

Ce document a été généré automatiquement le 19 avril 2019

\section{(c) (i) (9)}

Les contenus de la RIEF sont mis à disposition selon les termes de la Licence Creative Commons Attribution - Pas d'Utilisation Commerciale - Pas de Modification 4.0 International. 


\title{
Les médiations du désir dans Les Soleils des indépendances d'Ahmadou Kourouma
}

\author{
Alexandre Calvanese
}

1 Dans la littérature critique aujourd'hui abondante consacrée à l'œuvre d'Ahmadou Kourouma, il est assez rare de rencontrer des allusions à la théorie mimétique de René Girard ${ }^{1}$. Et pourtant les principes que Girard a montrés chez les romanciers analysés dans Mensonge romantique et vérité romanesque sont partout à l'œuvre dans l'univers de l'écrivain ivoirien. Il suffit de prendre en examen son premier roman, Les Soleils des indépendances, pour se rendre compte que Kourouma choisit comme « foyer narratif $»^{2}$ des personnages qui flottent entre le domaine de la médiation externe (où le modèle est trop éloigné du sujet pour être son rival) et celui de la médiation interne (où la distance entre sujet, objet du désir et médiateur s'est réduite au point de transformer l'émulation en antagonisme) pour en arriver enfin à la double médiation, où « le sujet devient le modèle de son modèle, et l'imitateur devient l'imitateur de son imitateur $»^{3}$. Ce qui nous intéresse ici est de montrer la manière particulière dont Kourouma a abordé le mécanisme mimétique dans son récit, car « le nombre de combinaisons mimétiques est infini, comme la façon de les exprimer » et chaque écrivain «exige une démonstration entièrement différente $»^{4}$. Le choix de cette démarche analytique présente un double avantage : elle rend plus transparentes, à l'intérieur du roman, les dynamiques qui gouvernent la formation et l'expression de l'identité dans la sphère individuelle (c'est le cas des deux personnages principaux, Fama et Salimata, sur lesquels nous nous attarderons le plus) mais aussi dans la dimension collective (l'arène politique de Togobala); en même temps, elle permet de considérer les affinités et les différences entre les exemples proposés tout en restant à l'intérieur du même cadre conceptuel - la théorie mimétique -, c'est-à-dire sans renoncer à la possibilité d'une lecture organique de l'œuvre.

2 Fama, le protagoniste du roman, est le dernier descendant de la dynastie des Doumbouya $\mathrm{du}$ Horodougou mais, en réalité, c'est un prince sans royaume. Si la colonisation l'a dépouillé des privilèges et des honneurs réservés à un chef traditionnel, les 
indépendances politiques ne lui ont rapporté « rien que la carte d'identité nationale et celle du parti unique $»^{5}$ : deux symboles que Fama méprise autant l'un que l'autre.

Nous trouvons par contre une image très représentative de la nostalgie que Fama ressent pour le prestige du temps passé : c'est le «coursier sans tache» (SI, p. 81) sur lequel Souleymane, le marabout fondateur de la dynastie des Doumbouya, arriva dans le Horodougou quelques siècles auparavant. Mais c'est aussi le «coursier blanc » (SI, p. 85) qui représente, pour le protagoniste, le bonheur de son enfance. L'image du cheval blanc peut donc évoquer deux différentes dimensions temporelles - l'origine de la dynastie d'un côté, l'histoire personnelle de l'autre -, mais, pour Fama, entre les deux plans il n'y a pas de véritable discontinuité logique, comme le montre cette séquence : "Son enfance! Son enfance! Dans tout il la surprenait, la suivait là-bas très loin à l'horizon sur le coursier blanc, il l'écoutait passer et repasser à travers les arbres, la sentait, la goûtait. Les exploits de ses aïeux le transportèrent » (SI, p. 85).

Évoquée pour la première fois au cours d'un voyage qui doit ramener Fama dans son village natal où il doit présider aux funérailles du cousin Lacina et en recueillir le titre de prince légitime du Horodougou, l'image du cheval revient à deux différentes reprises. La première est un rêve que Fama fait pendant sa captivité dans la prison de Mayako : «À califourchon sur un coursier blanc, Fama volait, plutôt naviguait, boubou blanc au vent, l'étrier et l'éperon d'or, une escorte dévouée parée d'or l'honorait, le flattait. Vrai Doumbouya! Authentique! Le prince de tout le Horodougou, le seul, le grand, le plus grand de tous " (SI, p. 141). La deuxième occasion se produit quand le roman est désormais arrivé à sa fin. Fama, blessé à mort par un caïman, délire: «Fama sur un coursier blanc qui galope, trotte, sautille et caracole. Il est comblé, il est superbe » (SI, p. 160).

5 Les analogies entre ces deux courts extraits sont évidentes, aussi bien que leur dépendance de l'image du fondateur de la dynastie. Le cheval blanc sur lequel Fama monte dans son rêve et dans son délire est le même cheval qu'a monté le marabout Souleymane, la figure légendaire que Fama interroge pour trouver une réponse à ses inquiétudes sur l'issue de son voyage ${ }^{6}$. Entre Fama et Souleymane, on retrouve donc un rapport identique à celui qui existait entre Don Quichotte et Amadis : le disciple qui tâche d'imiter son modèle. Une tentative d'émulation qui évolue jusqu'à frôler une impossible usurpation : Fama se substitue à Souleymane sur le cheval blanc car l'ambition secrète du sujet est toujours d'être comme le modèle.

6 Ce médiateur, lointain comme une divinité, « répand une lumière diffuse sur une surface très vaste $»^{7}$. Il ne désigne pas un objet en particulier, mais il a le pouvoir de modifier la perception que le sujet a du monde. C'est ainsi que, quand Fama arrive à Togobala, il découvre un paysage pour lui tout à fait inattendu : «Au nom de la grandeur des aïeux, Fama se frotta les yeux pour s'assurer qu'il ne se trompait pas. Du Togobala de son enfance, du Togobala qu'il avait dans le cœur il ne restait même plus la dernière pestilence du dernier pet » (SI, p. 85, nous soulignons). Ce moment de profonde mais temporaire désillusion a été soigneusement préparé par l'auteur, car jusqu'à cet instant-là, le village a été évoqué exclusivement à travers les souvenirs de Fama, principal point focal de la narration: « Ah! nostalgie de la terre natale de Fama! Son ciel profond et lointain, son sol aride mais solide, les jours toujours secs. Oh! Horodougou! tu manquais à cette ville et tout ce qui avait permis à Fama de vivre une enfance heureuse de prince manquait aussi (le soleil, l'honneur et l'or)»(SI, p. 20). Malheureusement la mémoire a été démentie par l'expérience directe et c'est bien de ce «décalage tragique [que] va découler le drame 
proprement dit $\|^{8}$ du héros. Ce n'est pas en vain que Fama a été peint dans le geste de se frotter les yeux, comme s'il venait de se réveiller après un long sommeil et qu'il n'arrivait pas à séparer rêve et réalité, tant et si bien qu'on l'a vu se diriger vers Togobala, le regard tourné en arrière vers le fondateur de la dynastie. La légende des Doumbouya, amplifiée au cours des siècles par les chants des griots, est devenue pour Fama le roman chevaleresque qui influence ses souvenirs et sa vision du monde.

7 Madeleine Borgomano a justement remarqué que l'espace, dans Les Soleils des Indépendances, est organisé selon « un système d'opposition binaire » qui « habite presque toute la littérature africaine francophone $»^{9}$ même si, sur un point, "le roman de Kourouma n'est pas conforme au modèle $»^{10}$. Dans le modèle, au village s'oppose la ville, et l'un et l'autre sont liés à des catégories, toujours en opposition, telles que Tradition et Modernité, ou Authenticité (africaine) et Corruption (occidentale). Dans le roman de Kourouma le village et la ville sont plus précisément associés à deux époques bien distinctes : avant et après les indépendances. Cette soudure spatiale et temporelle a une aura positive dans un cas (village-passé) et négative dans l'autre (ville-présent). Kourouma enrichit d'éléments nouveaux cette dichotomie. À travers le regard de Fama, dont nous écoutons les manifestations d'irritation sous la forme du discours indirect libre, la ville apparaît perpétuellement sous la pluie, envahie par l'eau, et le personnage subit cette situation comme s'il était victime d'un châtiment divin sous forme de siège, de pestilence ou de catastrophe naturelle :

Le soleil! le soleil! le soleil des Indépendances maléfiques remplissait tout un côté $\mathrm{du}$ ciel, grillait, assoiffait l'univers pour justifier les malsains orages des fins d'après-midi (SI, p. 13).

À droite, du côté de la mer, les nuages poussaient et rapprochaient horizon et maisons. À gauche les cimes des gratte-ciel du quartier des Blancs provoquaient d'autres nuages qui s'assemblaient et gonflaient une partie du ciel. Encore un orage! (SI, p. 19)

L'orage était proche. Ville sale et gluante de pluies ! pourrie de pluies ! (SI, p. 20) Les premières gouttes mitraillèrent et se cassèrent sur le minaret. [...] Le tonnerre cassa le ciel, enflamma l'univers et ébranla la terre et la mosquée. Dès lors, le ciel, comme si on l'en avait empêché depuis des mois, se déchargea, déversa des torrents qui noyèrent les rues sans égouts. Sans égouts, parce que les Indépendances ici aussi ont trahi, elles n'ont pas creusé les égouts promis et elles ne le feront jamais; des lacs d'eau continueront de croupir comme toujours et les nègres colonisés ou indépendants y pataugeront tant qu'Allah ne décollera pas la damnation qui pousse aux fesses du nègre. Bâtards de fils de chien! Pardon! Allah le miséricordieux pardonne d'aussi malséantes injures échappées à Fama dans la mosquée ! (SI, p. 24).

Comme pour souligner l'association très étroite que Fama établit entre la ville, la pluie et l'époque des indépendances, voici une similitude où l'orage, au lieu de se renverser sur la première comme dans les exemples ci-dessus, est comparé à la dernière : « Les soleils des Indépendances s'étaient annoncés comme un orage lointain » (SI, p. 22), tandis que, peu après, la pluie devient le symbole d'une existence malheureuse : « Fama devait prier pour détourner, écarter une vie semblable à une journée à l'après-midi pluvieux » (SI, p. 27).

Du côté du village, les éléments antithétiques à l'isotopie ville-indépendance-pluie sont tout aussi évidents : « Les souvenirs de l'enfance, du soleil, des jours, des harmattans, des matins et des odeurs du Horodougou balayèrent l'outrage et noyèrent la colère » (SI, p. 20). Au cours du voyage vers Togobala, le soleil devient significativement l'élément prédominant du décor : «Le soleil venait de pointer et s'empressait de fondre et balayer les nuages avant de monter plus haut et faire régner un vrai jour d'harmattan» (SI, p. 70). 
La répétition du verbe balayer, employé au sens figuré à l'intérieur de deux structures syntaxiques symétriques qui associent, dans la position de sujet, «les souvenirs de l'enfance » avec « le soleil », et dans la position de complément " outrage » et «nuage », rend le parallèle encore plus évident: le soleil balaye les nuages comme les souvenirs d'enfance balayent les outrages et la colère. Enfin, même après avoir constaté personnellement la modestie de son héritage et en général l'état de misère et de retard du village, Fama ne cesse d'éprouver une fascination enfantine devant des «tourbillons de vent, de poussière et de feuilles mortes [...] car dans son cœur remontaient les joies des bons harmattans de son enfance » (SI, p. 101).

10 Cette structure binaire comprend un autre couple d'éléments antithétiques : stérilité et fertilité. La première est la cause de violentes disputes et d'accusations réciproques entre Fama et sa femme Salimata. L'homme compare sa femme à " un grand fleuve » (SI, p. 25) dans lequel se noient les "grains sélectionnés " qu'il a déposés. Pour Fama l'eau est synonyme de stérilité tandis que le soleil et l'harmattan représentent la vie. Mais il suffit de rappeler la comparaison que Salimata employait pour se décrire «femme stérile comme l'harmattan » (SI, p. 28) pour constater que le même élément peut assumer une connotation de signe opposé et devenir ainsi le point convergeant de perspectives conflictuelles. Pour Fama, c'est la vie en ville qui s'éteint sous la pluie, entre misère et stérilité. Au contraire, le retour à Togobala pour les funérailles du cousin coïncide, pour le héros, avec la possibilité de prendre comme deuxième épouse la jeune veuve Mariam: une femme qui a déjà eu « un ou deux enfants » (SI, p. 76) et dont le ventre est capable, selon le sorcier Balla, de " porter douze maternités » (SI, p. 108).

11 La logique d'opposition binaire de Fama s'élargit donc comme une spirale qui absorbe tout avant de séparer ce qui offense de ce qui honore, ce qui repousse de ce qui accueille, ce qui provoque la rage de ce qui suscite l'admiration, en deux mots : l'Enfer du Paradis. Cependant Enfer et Paradis ne sont pas deux configurations cristallisées, définies à jamais, mais plutôt le produit d'une transfiguration qui se ressent du temps qui passe mais aussi du médiateur du désir. Fama idéalise le village à travers le filtre de la nostalgie de son enfance et de la légende des Doumbouya, et maudit la ville à travers celui de la rancune. Si nous relisons les passages que Kourouma consacre au passé de Fama nous découvrons que l'Enfer naît comme Paradis perdu ${ }^{11}$.

À l'époque du mouvement anticolonialiste, Fama, qui était un riche commerçant, avait cru pouvoir récupérer le rôle politique dont les Français et le cousin Lacina l'avaient privé en l'empêchant de succéder à son père sur le trône du Horodougou. À cette fin, il « s'était débarrassé de tout: négoces, amitiés, femmes» (SI, p. 22), pour se venger de cinquante ans de domination étrangère et d'humiliation personnelle. Mais une fois l'indépendance acquise, Fama fut jeté « comme la feuille avec laquelle on a fini de se torcher » (Ibidem). Dépourvu des compétences nécessaires pour aspirer aux rôles les plus prestigieux, Fama se trouva aussi exclu du partage des fonctions richement récompensées au sein du parti ou des coopératives. Et pourtant, remarque de manière sarcastique le narrateur, « il y avait quatre-vingts occasions de contenter et de dédommager Fama qui voulait être secrétaire général d'une sous-section du parti ou directeur d'une coopérative. Que n'a-t-il pas fait pour être coopté ?» (SI, p. 22). Dans le présent de la narration, Fama méprise les indépendances et leurs emblèmes, mais c'est seulement après les avoir obstinément courtisés. Comment expliquer cette incohérence avec la logique d'opposition binaire?

Quand il pense à son passé de grand commerçant, Fama éprouve le regret de s'être battu et d'avoir tout perdu pour une guerre inutile. Au moins les Français avaient favorisé le 
commerce, estime Fama, tandis que les Indépendances l'ont suffoqué! Voilà des réflexions qui montrent un certain opportunisme et qui portent atteinte à l'image de Fama, symbole d'une époque sur son déclin. Ce n'est pas le héros romantique, l'idéaliste tout d'une pièce qui se bat pour ses idées, mais l'opportuniste qui lutte pour s'assurer une place au soleil dans la nouvelle nomenclature politique et économique et qui regrette l'époque coloniale parce qu'elle était plus propice à ses affaires. Voilà pourquoi, selon Borgomano, Les Soleils des indépendances n'adhère pas au modèle binaire qui caractérise la littérature africaine de l'époque ${ }^{12}$. Kourouma nous propose un héros moderne, un personnage complexe qui oscille entre un idéalisme borné et des appétits vénaux : d'un côté le riche commerçant qui abandonne ses affaires parce qu' « un fils légitime des chefs devait de tout son être participer à l'expulsion des Français » (SI, p. 47), de l'autre un prince sans royaume qui a vu dans les indépendances un espoir de revanche mais qui a été rejeté par le nouveau monde et ses nouveaux maîtres ${ }^{13}$. Voilà son véritable Enfer : avoir tout essayé pour gagner le centre de la scène et se voir ignoré, sinon repoussé, par le public. Les quatre-vingts occasions perdues pour le dédommager sont autant de promesses qui ont été trahies, mais aussi autant de rivaux, tous confondus dans la même rancune, qui ont relégué Fama dans les coulisses. La transition entre le passé et le présent, la tradition et la modernité, l'époque de la chefferie et celle des indépendances se manifeste visiblement à travers ce glissement de la médiation externe à la médiation interne. La divinité de la dimension villageoise est Souleymane, trop lointain pour représenter un rival. En ville, dans l'ère des indépendances qui a remis en question les hiérarchies sociales traditionnelles, le médiateur se multiplie et se rapproche dangereusement et la contagion du désir mimétique est si répandue que « tout individu peut devenir le médiateur de son voisin sans comprendre le rôle qu'il est en train de jouer $»^{14}$.

La capitale, cœur de l'époque des indépendances qui a " oublié et jeté aux mouches » (SI, p. 22) Fama, devient l'emblème de la damnation du prince déchu. Toutefois, l'Enfer urbain garde toujours au fond un élément d'attraction irrésistible, c'est le modèle-obstacle contre lequel le sujet n'arrête pas de buter. Sans repérer cet aspect, il est difficile d'expliquer la décision de Fama de retourner dans la capitale alors qu'au village il a finalement tout ce qui lui manque en ville ${ }^{15}$ : «Qu'allait-il chercher ailleurs? Il avait sous ses mains, à ses pieds, à Togobala, l'honneur (membre du comité et chef coutumier), l'argent (Balla et Diamourou payaient) et le mariage (une jeune femme féconde en Mariam). Pourquoi tourner le dos à tout cela pour marcher un mauvais voyage? ? (SI, p.120). La question, purement rhétorique, montre la vacuité de tout désir né de la compétition: la conquête de l'objet convoité pendant de longues années n'apporte aucune réelle satisfaction ni aucun changement dans l'existence du héros ${ }^{16}$. Fama fera son retour en ville où il se lancera à corps perdu dans les palabres clandestines qui annoncent un complot contre le gouvernement. Son seul espoir est d'assister à la chute du pouvoir qui l'a repoussé :

Le pays couvait une insurrection. [...] On parlait de complots, de grèves, d'assassinats politiques. Fama exultait. Il rendait visite à ses anciens amis politiques, ses compagnons de l'époque anticolonialiste. Ceux-ci ne dissimulaient pas leurs soucis, ils avaient tous peur. Fama aimait les entendre dire que tout pouvait tomber sur le pays d'un instant à l'autre: les incendies, le désordre, la famine et la mort. Et au fond Fama souhaitait tout cela à la fois. (SI, p. 127). ruine collective à la victoire du rival détesté, de la mobilisation de l'être au service du 
néant. L'objet, quelle que soit la querelle, a disparu de l'horizon. Fama a un compte à régler avec l'idole qui l'a repoussé et il espère que le pays sera ravagé par un orage inattendu. Kourouma, à travers son personnage, nous montre de quelle façon le désir mimétique arrive à déclencher d'épouvantables tempêtes dans le verre d'eau de notre conscience malheureuse.

Salimata semble occuper le pôle antithétique par rapport à $\mathrm{Fama}^{17}$. Autant lui est incapable de trouver sa place et son rôle dans la société, autant elle est concrète et entreprenante. C'est bien elle, qui se dépense très tôt le matin entre le ménage et le travail en ville, le pilier du couple, le moteur productif de l'économie familiale. Cependant cette lecture cache plus de choses qu'elle n'en révèle. En effet, Fama et Salimata partagent la même attitude à lire la réalité avec les lunettes déformantes du désir mimétique et le personnage de Salimata représente sans aucun doute l'un des meilleurs exemples pour apprécier la dynamique de la médiation interne dans Les Soleils des indépendances.

Pour des raisons différentes, les deux personnages cultivent le même regret, c'est-à-dire l'absence d'un enfant, dont ils s'attribuent réciproquement la responsabilité. Pour l'un et pour l'autre, cette absence constitue un manque radical. Pour Fama, cela représente la fin de la dynastie des Doumbouya dont il est le dernier rejeton et dont il ne voudrait pas être la dernière goutte d'eau. Pour Salimata, la maternité est un état symbolique, une pensée qui l'accompagne nuit et jour et qui la pousse à essayer toutes sortes de remèdes pour tomber enceinte. Mais quand Salimata ne doit pas renvoyer à Fama l'accusation de stérilité, elle se croit réellement condamnée à un destin de "femme stérile comme l'harmattan et la cendre» (SI, p. 28). L'échec de ses ambitions est le produit d'une faiblesse ontologique qui la pousse à voir dans les autres des personnes meilleures qu'elle ${ }^{18}$. Son médiateur du désir est la femme épouse et mère de famille qui s'élève en idéal absolu de féminité: «Ce qui sied le plus à un ménage, le plus à une femme : l'enfant, la maternité qui sont plus que les plus riches parures, plus que la plus éclatante beauté ! À la femme sans maternité manque plus que la moitié de la féminité »(SI, p. 43-44).

Ce n'est pas l'horloge biologique qui bat la mesure du désir mais le prestige social d'une condition qui fait briller la femme plus que n'importe quelle parure. Dans une société patriarcale qui réserve à la femme un rôle éminemment servile (Salimata travaille toute la journée pour nourrir Fama) et de rares occasions pour être protagoniste, la maternité devient la substance de la féminité. Ce modèle de femme exerce sur Salimata une puissance extraordinaire, comparable à celle qui faisait voir à Don Quichotte le heaume de Mambrin là où il n'y avait qu'un plat à barbe :

Et les pensées de Salimata, tout son flux, toutes ses prières appelèrent des bébés. Ses rêves débordaient de paniers grouillants de bébés, il en surgissait de partout. Elle les baignait, berçait et son cœur de dormeuse se gonflait d'une chaude joie jusqu'au réveil. En plein jour et même en pleine rue, parfois elle entendait des cris de bébés, des pleurs de bébés. Elle s'arrêtait. Rien : c'était le vent qui sifflait ou des passants qui s'interpellaient. Un matin, elle rinçait les calebasses; sous ses doigts elle sentait un bébé, un vrai bébé. Elle le baigna, il pleurait en gigotant. Elle le porta dans la chambre et ouvrit les yeux. Rien : une louche dure et cassante. Et Salimata debout avec ses hontes et ses désespoirs. Une nuit, dans le lit, un bébé vint se coller à Salimata et se mit à la téter, les succions ont brûlé les seins gauche et droit, elle le tâta, tout chaud, tout rond, tout doux. Elle alluma la lampe : envolé, transformé en mortier de cuisine. Qui pouvait avoir introduit ce mortier? Salimata s'en doutait et les sorciers le relevèrent, le confirmèrent : c'était le génie de fatalité qui la hantait au village, qui l'avait rejointe dans la capitale. Il aimait Salimata, ne la quittait 
jamais. Les effets de cette assiduité éclatèrent rapidement: le génie engrossa Salimata! (SI, p. 44).

19 Vue, toucher et ouïe participent à la construction de la même illusion et sa durée limitée ne la rend pas moins significative que celle du chevalier de La Mancha. La puissance de transfiguration est exactement la même et semble suggérer une allusion bien précise : la louche et le mortier ne seraient-ils pas les doubles intertextuels du plat à barbe et des moulins de Don Quichotte ( $^{19}$ Cela dit, Kourouma est très habile dans l'alternance des différents points de vue de ses personnages et, après avoir poussé avec une mesure flaubertienne la balançoire d'illusions et d'amertumes où oscille Salimata, le voici changer rapidement de focalisation pour offrir au lecteur l'interprétation du mystère selon la sagesse populaire: les continuelles métamorphoses de l'objet convoité sont l'œuvre du génie, ce même esprit de la montagne auquel, quelques pages auparavant, un " on » indéfini avait attribué le viol dont une jeune Salimata avait été victime au village après son excision. Une persécution qui se prolonge dans la dimension urbaine jusqu'à ce que Salimata tombe enceinte.

Évidemment Kourouma ne croit pas aux génies des légendes populaires mais, en revanche, il cultive une passion très vive pour les mythologies individuelles qu'engendre toute relation mimétique et dont l'évolution de la grossesse de Salimata constitue probablement l'exemple le plus évident de son roman :

Qu'importe qu'après que tout fût tombé, se fût envolé, le docteur ait appelé cet état " une grossesse nerveuse " et les Malinkés "une grossesse de génie »! Salimata avait été heureuse des mois et des mois ; elle avait exulté ; elle avait été enceinte, avait eu un ventre et tout ce qui apparaît chez la femme qui attend. Elle s'était présentée à la maternité, elle avait été examinée et reconnue en grossesse, inscrite sur le registre des enceintes du quartier.

Pendant des mois, comme toutes les femmes en grossesse du quartier, cuillère et carnet à la main, Salimata avait monté la rue 5 , traversé une ou deux concessions avant l'avenue 8, puis le grand marché, avait salué les passants, leur avait dit qu'elle marchait vers le dispensaire pour avaler la cuillerée de potion des enceintes et les autres s'étaient empressés de féliciter, de prodiguer des bénédictions pour une bonne délivrance, un enfant de valeur, et avaient loué Allah d'avoir payé les bienfaits, la bonté et les prières de Salimata par une maternité.

Cela continua des mois et des mois, puis un an sans accouchement! Deux ans. Rien ! Petit à petit le ventre baissa et tout ce qui fait la femme enceinte dépérit et disparut. Ce qui est malheureux dans ce genre de choses, c'est la honte subséquente. Une honte à vouloir fendre le sol pour s'y terrer! Après des mois de grossesse sans avortement, sans accouchement, il faut sortir comme les autres, voir et parler aux autres, et rire aux gens. Evidemment les questions égratignent et embarrassent les gorges des interlocuteurs, on le voit. Alors, chaque fois on devient quelque chose, quelque chose de différent qui craint tout le monde... (SI, p. 44-45).

Kourouma propose ici deux points de vue antithétiques - celui de la médecine et celui de la sagesse populaire - pour revenir à la seule chose qui l'intéresse vraiment : l'expérience individuelle de son personnage. L'image de Salimata qui berce les ustensiles de cuisine est la dramatisation de cette forme de psychonévrose nommée grossesse hystérique, bien marquée par la triple métonymie qui décrit l'obsession du personnage : «Et les pensées de Salimata, tout son flux, toutes ses prières appelèrent des bébés ${ }^{20}$. La grossesse est pour Salimata la lettre de créance indispensable pour entrer dans un cercle exclusif qui reçoit ses membres tous les matins à la maternité du quartier : une fois sa demande d'admission agréée, elle peut être accueillie parmi les autres femmes enceintes avec les bénéfices symboliques (la «cuillerée de potion »), les vœux et les hommages qui lui sont dus. 
Salimata exulte parce qu'elle reconnaît sur elle les signes qu'elle voyait sur les autres femmes, mais malheureusement pour elle il ne s'agit que de la somatisation d'un désir jamais réalisé. Plus que n'importe quel autre héros kouroumien, Salimata incarne le paroxysme du désir mimétique qui, à son zénith, est capable de transformer le manque de l'objet convoité en des symptômes physiologiques qui simulent sa présence et rendent le sujet identique à son médiateur. À travers le désir exceptionnel de ce personnage, Kourouma montre ainsi toute l'influence de la dynamique mimétique dans la vie quotidienne.

22 Le moment de la chute est symétrique à celui du triomphe. La disparition du ventre est pour Salimata une honte qui a la même valeur qu'une expulsion du cercle des femmes enceintes. D'ailleurs l'exclusion-expulsion d'un cercle restreint semble une constante de la vie de ce personnage, car déjà l'excision, « qui aurait dû la faire entrer dans un groupe social, l'en a exclue $»^{21}$. Voilà, finalement, la véritable disgrâce. Kourouma ne parle pas de la déception de Salimata pour l'enfant qui ne naîtra jamais, mais de son malaise à l'idée de devoir se justifier devant le monde. La disparition de l'objet convoité a une importance tout à fait relative ${ }^{22}$. La cause réelle du malheur du personnage est la certitude de ne pas être à la hauteur de l'idole que le sujet lui-même s'est proposé comme modèle. Une idole qui, au début de la troisième partie du roman, acquiert incontestablement le profil de rival avec le personnage de Mariam, la veuve du cousin Lacina que Fama a choisie comme deuxième femme et amenée en ville avec lui. L'atout de Mariam est le fait d'avoir déjà eu des enfants, ce qui provoque l'envie de Salimata et déclenche la première dispute entre ces deux femmes :

À chaque réveil, Salimata regardait le ventre de la coépouse, et le ventre semblait pousser. Oui, il poussait! Salimata devint jalouse, puis folle et un matin elle explosa, injuria. Les deux coépouses comme deux poules s'assaillirent, s'agrippèrent l'une au pagne de l'autre. Mariam voulait coûte que coûte tomber le pagne de Salimata afin que chacun vit « la matrice ratatinée d'une stérile » et Salimata dévêtir Mariam afin que tout le monde reconnût « la chose pourrie et incommensurable d'une putain » ( SI, p. 125-126).

Bien avant de se disputer Fama, les deux femmes luttent pour quelque chose qui est, à leurs yeux, encore plus essentiel. Cette scène complète le récit de la grossesse hystérique car dans ce cas l'obsession de Salimata se projette sur la rivale. Mariam n'est pas enceinte mais Salimata voit son ventre grandir : voici une deuxième grossesse imaginaire! Le regard mimétique de Salimata voit en Mariam le modèle qui dit « imite-moi » et, en même temps, l'obstacle qui empêche cette émulation car elle est bien sa coépouse. L'auteur, passant de la focalisation interne ${ }^{23}$ («Oui, il poussait ! » est indiscutablement une pensée de Salimata rapportée à travers le discours indirect libre) à la focalisation externe («Les deux coépouses comme deux poules s'assaillirent»), rend le mécanisme mimétique évident dans toute son implacabilité : Salimata éprouve pour Mariam une si forte envie qu'elle en arrive d'abord à l'imaginer enceinte, ensuite à l'agresser, provoquant chez sa rivale une réaction identique à la sienne. Il suffit de repérer cette alternance entre le point de vue du personnage et le point de vue du narrateur sur le personnage pour saisir le décalage entre le mensonge romantique, qui attribue toujours à l'autre la cause de son malheur, et la vérité romanesque qui, dans ce cas, éclaire la réciprocité de la violence mimétique.

24 La médiation double, ou réciproque ${ }^{24}$, est largement diffuse aussi dans l'univers de Togobala, l'atelier politique dans lequel Kourouma a élaboré des personnages qu'il affinera vingt ans plus tard dans Monnè, outrages et défis. Un des thèmes centraux de toute 
sa création littéraire est la vacuité qui nourrit la rivalité entre factions et partis politiques.

Pour le parti unique de Togobala, le retour du réactionnaire Fama, venu pour reprendre ce qui lui a été enlevé, est une manne céleste car ses membres peuvent crier « l'ennemi est aux portes ». Mais c'est aussi le parti légitimiste - animé par le griot Diamourou et par l'ancien esclave, féticheur et chasseur Balla ${ }^{25}$ - qui bénéficie de son retour pour trouver une nouvelle vigueur :

Diamourou et Balla avaient enfumé le village d'autres plus insolentes paroles. Leur maître s'entraînait à tordre le cou aux Indépendances, au parti unique et à tous les comités. « Il y avait de la contre-révolution, de l'authentique réaction à Togobala ! » estimèrent le comité et son président, et ils le crièrent. Le sous-préfet, le secrétaire général, le gouverneur, le parti unique exultèrent (depuis des mois il n'y avait plus de réactionnaire à dépister), et dégainèrent, prêts à décapiter dans le nid l'horrible contre-révolutionnaire (SI, p. 109, nous soulignons).

Tout le monde s'agite dans le seul but de faire monter la température du conflit et de trouver ainsi une bonne raison pour s'engager davantage dans un affrontement qui n'a pas d'enjeu réel. Les ambitions de Fama et de ses alliés - la réhabilitation de la chefferie dans un village décrépit à la périphérie de l'histoire - sont aussi ridicules que les alarmes hystériques lancées par ses adversaires, les soi-disant révolutionnaires qui pourtant exultent pour le retour de la réaction, car ils en ont désespérément besoin pour définir leur identité anti-réactionnaire. La rivalité est tellement dépourvue de contenu qu'à la fin, après d'interminables discussions mises en scène dans l'unique intention d'entretenir le public du village, la situation demeure inchangée : «Fama resterait le chef coutumier, Babou le président officiel» (SI, p. 113). Chaque faction garde son champion et son ennemi, tous les deux indispensables pour définir l'identité et resserrer des rangs un peu effilochés. Balla et Diamourou, par exemple, en dehors de l'arène politique, sont des rivaux acharnés qui se tolèrent à peine, apparemment à cause de la religion - le premier est féticheur, l'autre est musulman. « Mais, sur tous les autres plans, Diamourou et Balla sont solidaires $»^{26}$ : ils partagent le rôle de témoins de la déchéance des Doumbouya aussi bien que l'astuce et l'opportunisme qui leur ont permis de traverser indemnes l'époque de la colonisation pour aborder celle de l'indépendance en position de privilège. Après avoir passé toute une vie au service de la famille Doumbouya, Diamourou et Balla mettent leurs substances à la disposition de Fama pour compenser sa détresse mais surtout pour faire revivre un rêve désormais évanoui face auquel le narrateur suggère au lecteur une certaine méfiance :

Au soir de leur vie les deux vieillards œuvraient à la réhabilitation de la chefferie, au retour d'un monde légitime. Malheureusement, Togobala, les Doumbouya et même le Horodougou ne valaient pas en Afrique un grain dans un sac de fonios. Qu'importe, ils y croyaient, ils s'y employaient (SI, p. 94).

Encore une fois Kourouma recourt, en trois courtes phrases, à l'alternance des regards pour dévoiler l'absence de perspective qui se cache derrière toute entreprise, comme la réhabilitation de la chefferie, inspirée par la rivalité mimétique, dans ce cas celle qui a lieu entre "révolutionnaires" et "légitimistes». Et à l'intérieur de ce dernier regroupement, l'impulsion émulatrice est tellement irrésistible que les deux faux rivaux, Balla et Diamourou, se retrouvent enfin sur la même route, persuadés que c'est le dernier prince légitime du Horodougou qui demande leur indispensable collaboration. Mais la chefferie est désormais morte, tout comme Togobala et la dynastie des Doumbouya. Ce qui survit est seulement un inépuisable esprit de compétition face à un médiateur qui est 
de plus en plus proche. La différence entre le marabout Souleymane et les adversaires politiques de Togobala ne tient, finalement, qu'à cet aspect. De même, les différences entre Fama et Salimata, ou entre ces deux derniers et Diamourou et Balla, sont beaucoup moins radicales si on les observe à l'intérieur d'une structure triangulaire qui est suffisamment flexible pour tenir compte du changement de position du modèle-rival et donc des différentes réactions (l'émulation ou l'admiration plutôt que la jalousie ou la rivalité) que celui-ci peut engendrer chez le sujet mimétique. D'ailleurs, c'est justement ce rapport ambigu avec le médiateur du désir qui est à la base des hallucinations ou des illusions dont les personnages qui peuplent le roman de Kourouma sont invariablement victimes, au niveau individuel aussi bien que collectif.

La lecture mimétique permet donc de déceler, dans Les Soleils des indépendances, une cohérence interne et une unité d'inspiration absolument remarquables, qui demandent à être vérifiées dans le cadre d'une relecture globale de l'œuvre romanesque d'Ahmadou Kourouma.

\section{NOTES}

1. À notre connaissance: M. Borgomano, Ahmadou Kourouma. Le "guerrier griot», Paris, L'Harmattan, 1998, p. 104 et 214; P. Soubias, "Les Soleils des Indépendances: la magie du désenchantement", Notre Librairie. Revue des littératures du Sud, n. 155-156, "Cahier spécial Ahmadou Kourouma : l'héritage ", juillet-décembre 2004, p. 148. Pour un aperçu des tendances de la critique sur Kourouma, voir C. Ndiaye, «Kourouma, the Myth: the Rhetoric of the Commonplace in Kourouma Criticism», Research in African Literatures, vol. 38, n. 2 (Summer, 2007), p. 95-108.

2. G. Genette, Figures III, Paris, Seuil, 1972, p. 203.

3. R. Girard, Les origines de la culture, [Desclée de Brouwer, 2004 ${ }^{1}$ ], Paris, Hachette Littérature, «Pluriel », 2009, p. 62.

4. Ibid., p. 228.

5. A. Kourouma, Les Soleils des Indépendances, Monnè, outrages et défis, En attendant le vote des bêtes sauvages, Allah n'est pas obligé, Quand on refuse on dit non, Le Diseur de vérité, Paris, Le Seuil, « Opus », 2010, p. 23. Toutes les citations du roman Les Soleils des Indépendances sont tirées de cette édition (dorénavant SI).

6. Mais, pour en revenir à la question de l'homogénéité entre deux plans temporels différents, on pourrait aussi bien affirmer, d'après P. Ngandu Nkashama (Kourouma et le mythe, Paris, Silex, 1985, p. 106), que ce sont les souvenirs de l'enfance qui « font [...] irruption dans la mémoire tourmentée de Fama, chaque fois qu'il lui faut dépasser une situation difficile ».

7. R. Girard, Mensonge romantique et vérité romanesque, [Grasset, 1961 ${ }^{1}$, Paris, Hachette, 1999, p. 102.

8. P. Ngandu Nkashama, op. cit., p. 106.

9. M. Borgomano, op. cit., p. 55.

10. Ibid., p. 56.

11. D'ailleurs le village aussi, sans devenir infernal, n'est plus qu'un Paradis perdu. Cf. ibid., p. 55. 
12. L'attitude ambiguë de Fama par rapport à la colonisation brise la dichotomie ville-village, car « chaque pôle est à la fois positif et négatif, la boussole est désorientée » (Ibid., p. 56).

13. Voir aussi A. Koné, «L'effet de réel dans les romans de Kourouma », Études françaises, vol. 31, n. 1, 1995, p. 17.

14. R. Girard, Mensonge romantique et vérité romanesque, op. cit., p. 118.

15. Selon M. Borgomano (op. cit., p. 56), « les mouvements de Fama dans la suite du roman montrent l'affolement d'un être dont le système de valeur est détruit, sans être remplacé par un autre ». À notre avis il s'agit plutôt de l'exaspération de la dynamique mimétique.

16. Ce n'est qu'à la fin du roman, après la captivité dans la prison de Mayako, que Fama vivra sa « conversion romanesque » et renoncera à tout désir mimétique. Cf. P. Soubias, op. cit., p. 148.

17. Voir M. Borgomano, op. cit., p. 63 et V. Bonnet, « Histoire du féminin, discours au féminin dans l'œuvre d'Ahmadou Kourouma ", Études françaises, vol. 42, n. 3, 2006, p. 112.

18. Voir l'interprétation du bovarysme proposée par Girard dans Mensonge romantique et vérité romanesque, op. cit., p. 79-80.

19. Le mimétisme en littérature se révèle aussi dans ce jeu de citations plus ou moins explicites, plus ou moins conscientes, qui a été d'ailleurs une source d'inspiration pour d'innombrables réflexions, articles et livres sur la nature intertextuelle du discours littéraire.

20. Nous soulignons.

21. E. Langlois, « Les soleils des Indépendances, roman de la stérilité ? », Présence francophone, n. 8, printemps 1974, p. 96.

22. "L'objet n'est qu'un moyen d'atteindre le médiateur. C'est l'être de ce médiateur que vise le désir ", R. Girard, Mensonge romantique et vérité romanesque, op. cit., p. 69.

23. M. Borgomano (op. cit., p. 60) observe que Salimata est le seul personnage, avec Fama, qui a droit à la focalisation interne et donc au « statut de Sujet, équivalent à celui du héros ».

24. R. Girard, Mensonge romantique et vérité romanesque, op. cit., p. 115-133.

25. Dans le deuxième roman, nous retrouvons un couple spéculaire à celui-ci dans les personnages de Diabaté et Fadoua.

26. J.-C. Nicolas, Comprendre "Les soleils des indépendances », Issy-les-Moulineaux, Les classiques africains, 1985, p. 66.

\section{INDEX}

Mots-clés : Girard (René), Kourouma (Ahmadou), Soleils des indépendances, médiation du désir, théorie mimétique, indépendances africaines, rivalité 